\title{
Nova técnica para sincronismo em tempo e freqüência de sinais OFDM baseada em reconhecimento de padrões
}

\author{
Rangel Arthur, Yuzo Iano, IEEE Member, Roger F. Larico, Luiz H. Bonani, Diogo G. Caetano, \\ Alexandre G. Silva
}

\begin{abstract}
Este trabalho propõe uma nova técnica para se obter o sincronismo de sinais baseados em OFDM (Orthogonal Frequency Divivion Multiplexing). Tal técnica é baseada na distância de Hamming generalizada, utilizada principalmente em métodos de reconhecimento de padrões. É realizada então uma comparação de complexidade e analisado desempenho entre as técnicas clássicas e a nova proposta diante de uma variedade de canais. A técnica proposta mostra-se robusta e apresenta baixa complexidade computacional.
\end{abstract}

Palavras-chave - Sincronismo, OFDM, Reconhecimento de padrões, TV Digital.

Abstract- This work aims new technique to synchronize signals based on OFDM (Orthogonal Frequency Divivion Multiplexing). This technique is based on generalized Hamming distance, mainly used in pattern recognition methods. A complexity comparison between the proposed and classical technics is achieved using several types of channels. The proposed method shows good performance and low computational complexity.

Index Terms - Synchronism, OFDM, Pattern Recognition, Digital TV.

\section{INTRODUÇÃO}

Sabe-se que um sinal OFDM oferece maior eficiência no uso do espectro e robustez para interferência intersimbólica. Além disso, o OFDM é mais eficiente ao combate de desvanecimento seletivo em freqüência e ruído impulsivo no domínio do tempo quando comparado à modulação de portadora única, como abordado em [1]. No entanto, apresenta desvantagens como sensitividade para se obter o desvio de freqüência entre os osciladores do transmissor e receptor, que dificulta o sincronismo do sinal recebido [2]. Para alcançar o melhor desempenho em sistemas de TV Digital, o sincronismo deve estar corretamente estabelecido.

Rangel Arthur e Luiz H. Bonani são professores de Tecnologia em Telecomunicações do Centro Superior de Educação Tecnológica (Ceset) da Unicamp, Limeira-SP, Brasil, fone: 55-19-2113-3492; e-mail: rangel, bonani@ ceset.fee.unicamp.br.

Yuzo Iano é professor do Departamento de Comunicações (DECOM) da Faculdade de Engenharia Elétrica e de Computação FEEC-UNICAMP; e-mail: yuzo@decom.fee.unicamp.br

Roger Larico e Diogo G. Caetano são alunos de Doutorado do DECOM da FEEC-Unicamp.

Alexandre G. Silva é professor de Ciências da Computação da UDESC.
Na recepção de um sinal OFDM, antes de demodular as portadoras, é necessário realizar dois tipos de sincronização. A primeira é destinada a encontrar os limites do símbolo OFDM (sincronismo temporal) que irá minimizar os efeitos de interferência entre símbolos (ISI - Inter Symbol Interference) OFDM. A segunda tem como objetivo achar a variação de freqüência (sincronismo de frequiência) das portadoras para que não ocorra interferência entre subportadoras (ICI - Inter Carrier Interference).

Em um sistema OFDM as portadoras são totalmente ortogonais somente se o transmissor e o receptor estiverem sincronizados em frequiência. Qualquer variação de freqüência resulta em ICI. Esse problema pode ser gerado pelo ruído de fase dos osciladores do transmissor e receptor. $\mathrm{O}$ ruído de fase modula aleatoriamente a fase do oscilador, resultando em uma variação de frequiência (jitter) que causa ICI e ISI no receptor OFDM. Ao contrário disso, para os sistemas de portadora única, o ruído de fase apenas degrada a relação sinal-ruído no receptor, pois as constelações perdem o foco. Desta forma, o sistema OFDM é altamente sensível a erros de sincronização em relação aos sistemas de portadora única.

Vários trabalhos têm sido propostos para diminuição de complexidade dos algoritmos de sincronismo, entre eles [36]. A diminuição de complexidade é um importante foco neste trabalho. Para o sincronismo temporal, o cálculo da correlação entre as regiões definidas pelo intervalo de guarda, conforme ilustrado na Fig.1, foi defendido em vários artigos, como em Palin [7]. Alguns deles abordam o processamento posterior aos vários pontos de máximos locais alcançados nessa fase, os trechos analisados são mais semelhantes, principalmente diante de ambientes com multicaminho. Este problema é mais acentuado em casos de ambientes de redes de frequiência única, abordado pelo mesmo autor em [8].

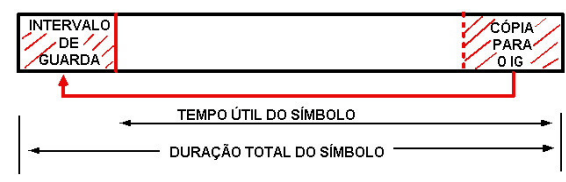

Fig. 1. Comparação de intervalos de guarda de um símbolo OFDM.

Uma importante característica na grande maioria dos receptores, diante da presença de multicaminho, é a capacidade do equalizador adaptativo somente recuperar o sinal se o ponto alocado de sincronismo temporal estiver dentro do intervalo de guarda. Este artigo está organizado da seguinte maneira. Na Seção II são apresentados os blocos 
comuns dos receptores OFDM, que são utilizados nesta etapa de processamento. Na Seção III, são discutidos alguns efeitos da falta de sincronismo. Nas seções IV e V, são descritas algumas fases comuns de vários algoritmos para sincronismo em tempo e freqüência. As técnicas propostas e as simulações são apresentadas nas seções VI e VII. O trabalho é finalizado com as conclusões relevantes.

\section{TÉCNICAS CLÁSSICAS DE SINCRONISMO TEMPORAL}

\section{A. MÉTODOS BASEADOS NA AUTOCORRELAÇÃO DE UM SÍMBOLO OFDM}

Um dos mais famosos métodos de autocorrelação é provavelmente o então definido método de Schmidl e Cox [9]. A recuperação de tempo de símbolo neste caso procura um símbolo de treinamento que tem duas metades idênticas no domínio do tempo. Tal símbolo deve ser enviado antes da transmissão de informação útil.

O processamento é realizado percorrendo o sinal recebido, obtendo a autocorrelação de duas partes, que compreendem metade do tamanho da FFT e procuram-se os valores máximos da saída do circuito de autocorrelação, como mostrado na figura a seguir.

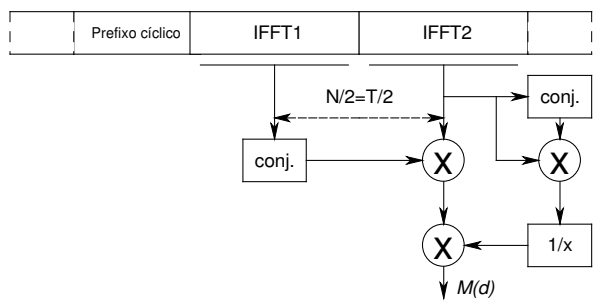

Fig.2. Princípio do circuito de sincronização baseado em autocorrelação proposto em [9].

A métrica $M$ deste sincronizador, que revela a similaridade entre dois trechos analisados do sinal recebido $r$, é calculada de acordo com a expressão

$$
M(d)=\frac{\left|\sum_{m=0}^{N_{c} / 2-1} r_{d+m}^{*} r_{d+m+N_{c} / 2}\right|}{\left(\sum_{m=0}^{N_{c} / 2-1}\left|r_{d+m+N_{c} / 2}\right|\right)} .
$$

sendo $N_{c}$ o número de subportadoras do símbolo OFDM e $d$ a posição inicial do vetor recebido $r$ para a análise.

A principal vantagem desta métrica é sua robustez perante desvios de frequiência e fading. Além disso, o sinal $r$ pode ser gerado por esquemas OFDM clássicos, no qual as duas metades idênticas são construídas através de sequiências pseudo-aleatórias (PN) nas frequiências pares, enquanto zeros são usados nas freqüências ímpares [9].

\section{B. MÉTODOS BASEADOS EM PREFIXO CÍCLICO}

No método anteriormente descrito, a sincronização exige informação adicional na forma de símbolos ou portadoras piloto de treinamento. Tal método é ideal em comunicação ponto-a-ponto, mas inviável em aplicações de radiodifusão.

Uma maneira de compensar a falta de informação de treinamento e se realizar o sincronismo temporal é usar o prefixo cíclico, que está presente na maioria dos sistemas OFDM. Um simples esquema é mostrado na Fig.3 a seguir.

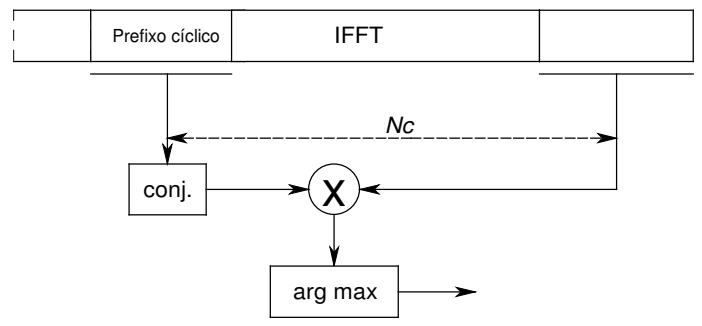

Fig.3. Princípio da sincronização baseada em prefixo cíclico.

Este esquema corresponde ao estimador ótimo no caso de canais AWGN. Assim, usando essa condição do sinal recebido e procurando estimar a máxima verossimilhança $\mathcal{E}_{M L}$ a partir de um atraso temporal, chega-se a solução

$$
\varepsilon_{M L}=\arg \max _{\varepsilon}(|\gamma(\varepsilon)|-\rho \phi(\varepsilon))
$$

onde $\mathcal{E}$ identifica o atraso temporal de análise do sinal

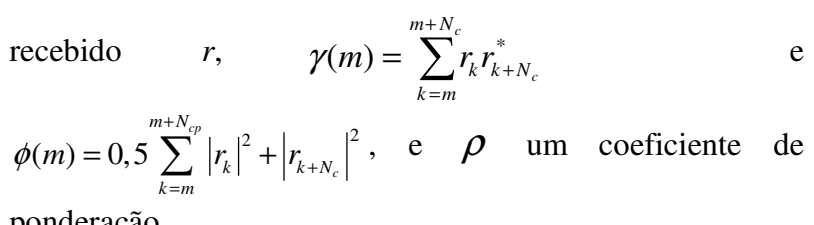
ponderação.

Este estimador de sincronismo possui as seguintes características: alta eficiência espectral por não usar pilotos; apesar da expressão relativamente simples, possui alta complexidade; pode realizar, dependendo do canal, estimação de desvios em tempo e freqüência. A principal desvantagem do estimador é que na presença de multicaminhos severos seu desempenho é reduzido. Neste caso, quanto maior o atraso do multicaminho, menor será a característica cíclica do sinal, que se torna nula para atrasos do tamanho do intervalo de guarda.

\section{TÉCNICAS CLÁSSICAS DE SINCRONISMO EM FREQÜÊNCIA}

\section{A. MÉTODOS BASEADOS NA AUTOCORRELAÇÃO DE UM SÍMBOLO OFDM}

Um dos primeiros métodos de autocorrelação proposto na literatura foi o então denominado método de Moose [10]. Neste método, uma seqüência inicial de treinamento, que consiste de dois símbolos OFDM idênticos sem IG, é usado. $\mathrm{O}$ método tem como premissa o fato dos dois símbolos OFDM estarem relacionados, considerando um desvio de freqüência $\Delta f_{c}$ e uma transmissão sem ruído, de acordo com a expressão 


$$
r_{l+1}=r_{l} e^{2 \pi j \frac{\Delta f_{c} N_{c}}{B}}
$$

com $B$ sendo a largura de banda do canal e $N_{c}$ o número total de portadoras.

Da expressão anterior, pode-se mostrar que o estimador de máxima verossimilhança (ML - Maximum Likelihood) do desvio de frequiência é dado por

$$
\hat{\Delta} f_{c}=\frac{B}{2 \pi N c} \tan ^{-1}\left\{\frac{\sum \operatorname{Im}\left(r_{l+1} r_{l}^{*}\right)}{\sum \operatorname{Re}\left(r_{l+1} r_{l}^{*}\right)}\right\} .
$$

$\mathrm{O}$ resultado anterior mostra que o ângulo de $r_{l+1} r_{l}^{*}$ é igual a $\frac{2 \pi \Delta f_{c} N_{c}}{B}$, como representado na figura a seguir.

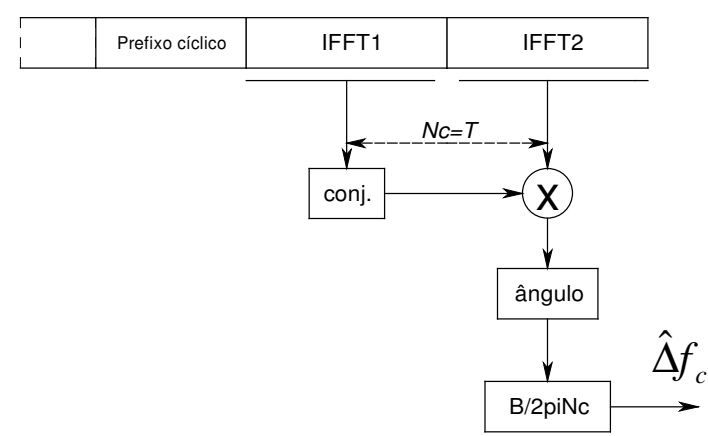

Fig.4. Princípio da autocorrelação Moose para sincronização em freqüência.

\section{B. MÉTODOS BASEADOS NO PREFIXO CÍCLICO}

De forma semelhante às propostas iniciais de sincronismo temporal, a proposta anterior para sincronismo em freqüência utiliza informação adicional de dois símbolos OFDM, o que é inviável em uma transmissão de TV. O mesmo princípio, descrito no IV.B, pode ser tomado para que tal estimação em freqüência possa ser aplicada. A Fig. 5 mostra esse procedimento, que é equivalente ao estimador ML da expressão (2).

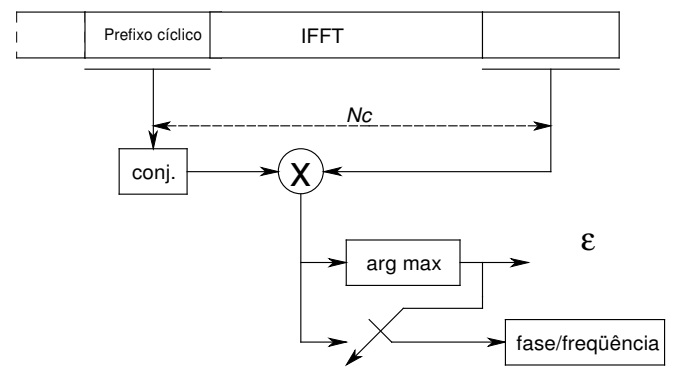

Fig.5. Princípio da autocorrelação Moose para sincronização em frequiência.

Novamente, a principal vantagem deste estimador é sua simplicidade. Por outro lado, é um método sensível a multicaminhos, e nesse caso é necessário utilizar sinais piloto.

\section{NOVA PROPOSTA DE SINCRONISMO TEMPORAL}

Na expressão (2) anterior é apresentado um estimador de sincronismo temporal que pode ser aplicado em uma situação real, sem o uso de seqüências de treinamento. Porém, o número de multiplicações respectivas ao termo $\gamma$ pode inviabilizar o sincronismo. Nesse caso, os deslocamentos temporais para se encontrar um ponto ideal, ainda mais diante de multicaminhos característicos de redes SFN, podem fazer com que o processo se torne excessivamente complexo computacionalmente.

Diante disso, passou-se a investigar um novo método para comparar trechos referentes ao intervalo de guarda (IG) ou prefixo cíclico (CP - Cyclic Prefix). Os trechos de IG, como mostrados na Fig. 3, são semelhantes, porém submetidos a diferentes tipos de degradações. Tal característica é comum em sistemas que procuram relacionar determinada amostra com outras de uma base de dados, como técnicas usadas em reconhecimento de padrões [11]. O reconhecimento de padrões é a ciência que trata da classificação e descrição de objetos, e pode ser aplicado em diversas áreas, como: científicas, industriais, médicas, agricultura, militar etc.

Podem ser citadas algumas grandes aplicações de reconhecimento de padrões como em áudio, para reconhecimento de voz, por exemplo, ou imagens, como de impressão digital e íris, colocadas na Fig. 6 apenas como ilustração.

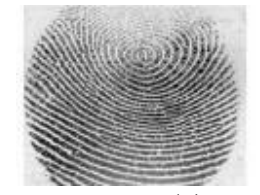

(a)

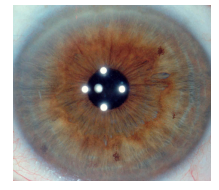

(b)
Fig.6. Ilustração de aplicações de reconhecimento de padrões (a) reconhecimento de impressão digital (b) reconhecimento de íris.

Dentre as principais técnicas usadas para reconhecimento de íris, podem ser citadas as wavelets [12,13], e, mais recentemente, a distância de Hamming [14]. Devido à complexidade de se realizar a transformada de wavelet, tal técnica foi preterida pela segunda opção.

A distância de Hamming, proposta inicialmente em 1950 [15] para implementação dos famosos códigos de Hamming, é então utilizada aqui para sincronismo temporal de símbolos OFDM. A comparação entre dois sinais para identificação de semelhanças pode ser aplicada para se procurar os respectivos trechos do intervalo de guarda dos símbolos, submetidos às degradações.

Inicialmente, a partir do sinal recebido, se obtém as informações do modo de transmissão (número de portadoras) e intervalo de guarda. A posição inicial do símbolo periódico do sinal, com certo grau de erro, é estimada a partir do intervalo de guarda no domínio do tempo, de maneira similar aos trabalhos de Prast e outros [16] e de Beek e outros [3].

O estimador baseado na distância de Hamming é proposto na Fig. 7, e tem como diferença uma grande diminuição drástica de complexidade. As multiplicações da variável $\gamma$ apresentada na Equação (2) são substituídas por simples subtrações. O bloco que contém as duas barras paralelas representa o módulo das subtrações. Além disso, é 
utilizados um parâmetro de entrada que diminui o número de iterações, com diminuição controlada de precisão.

Seja $r(k)$ o sinal recebido, pode-se correlacionar regiões respectivas ao IG da seguinte forma:

$$
k_{e s t}=s \cdot \arg \min _{k} \sum_{i=0}^{L-1}|r(i+k * s)-r(i+N+k * s)|
$$

sendo $k_{\text {est }}$ o ponto estimado temporalmente para início do símbolo.

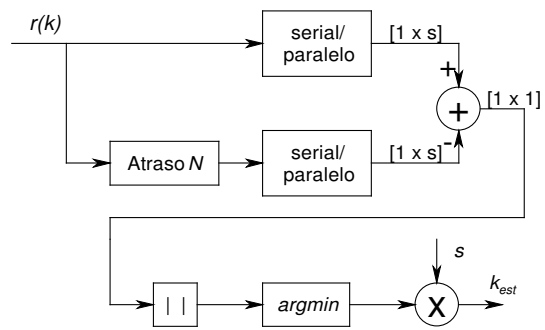

Fig.7. Bloco que representa o correlator.

Para obter o melhor desempenho do somatório apresentado na expressão (7), é adicionado um parâmetro $s$, que acelera o salto de amostras. Dado que $s=(N+L) / k$, sendo que $k$ pode variar de $L$ até $(N+L)$ amostras, sendo $s$ a quantidade de iterações. Deste modo, quando $s=1$, se obtém uma correlação amostra por amostra. Para grandes valores de $s$, será obtida uma estimativa através de um menor número de iterações, porém com o erro maior em amostras. No caso específico, o valor máximo para se obter uma estimativa correta com pouca precisão é de $s=L$, dado que a região de comparação é o intervalo de guarda de tamanho $L$. Na Fig.8 a seguir é mostrado o comportamento do pico da correlação $k_{\text {est }}$ invertido.

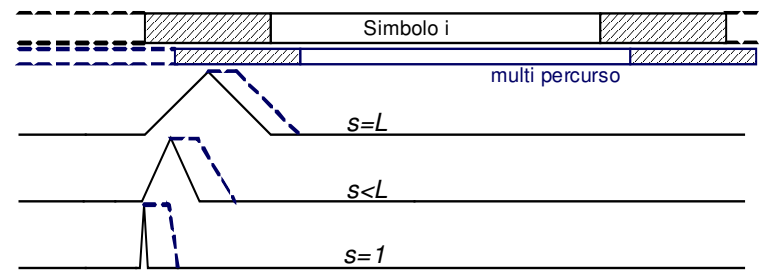

Fig.8. Comportamento do pico do correlator baseado na mínima distância (invertido).

Podem surgir problemas para se encontrar o pico da correlação em canais de atrasos muito longos, como discutido em [8]. Nesses casos, o pico encontrado no caminho com maior atraso não deve ultrapassar o tamanho do intervalo de guarda.

O sincronizador utilizado é mostrado na Fig.9. O sinal dentro do bloco de sincronismo é convertido da forma serial para paralela em até $s$ saídas por conversor, sendo o parâmetro $s$ responsável em diminuir a quantidade de iterações.

O sincronismo de símbolo mostrado na figura anterior é realizado através de $k_{e s t}$. Este parâmetro é encontrado inicialmente, porém com certo erro, dependente do parâmetro $s$. Se $s$ for maior que uma unidade, será criada uma região de início de símbolo em vez de um único ponto, como mostra a Fig.10. Tendo como trecho um erro máximo de $s$ amostras para direita ou $s$ amostras para esquerda. Esta região é considerada então como a "região de início" de símbolo e o sinal é considerado sincronizado em tempo. Isto pode ser adotado porque se tem a garantia de que o sinal sempre estará dentro do intervalo de guarda, como mostrado nos casos da Fig.8, possibilitando posteriores refinamentos.

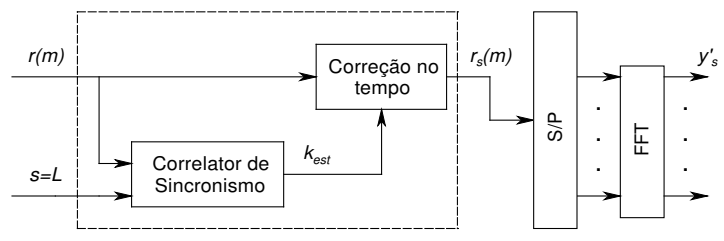

Fig.9. Implementação do correlator dentro do sistema.

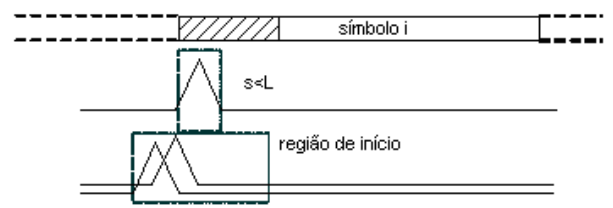

Fig.10. Região de início de símbolo.

\section{NOVA PROPOSTA PARA SINCRONISMO EM FREQÜÊNCIA}

A partir do sincronismo temporal do sinal OFDM, escolhido dentro do intervalo de guarda, são utilizados sinais pilotos espalhados para o refinamento e obtenção da janela FFT perfeita.

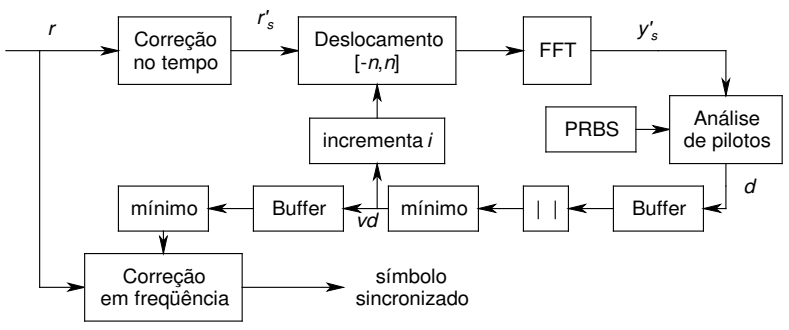

Fig. 11. Esquema de ajuste em freqüência através de pilotos espalhadas.

O algoritmo proposto, cujo esquema aparece na Fig. 11, faz a procura dos pilotos e tenta anulá-los. Para isso, utilizam-se as sequiências pseudo-aleatórias (PRBS) invertidas dos padrões e busca-se uma função constante. $\mathrm{O}$ ponto de sincronismo escolhido será aquele em que a função constante mais se aproximar de zero, conforme o algoritmo seguinte.

\section{Algoritmo de Refinamento}

1. Armazenar, a partir do ponto obtido no estágio de sincronismo temporal, $n$ amostras à esquerda e $n$ amostras à direita do sinal recebido, na variável $y_{s}^{\prime}$, sendo $n<\mathrm{IG}$;

2. Gerar a sinalização PRBS para os quatro padrões de símbolo, fixados pela norma ISDB-T [17]. O vetor PRBS1 será constituído assim de valores $+1 \mathrm{e}-1$;

3. Os sinais gerados no passo2 são multiplicados 
pontualmente pelo sinal $y_{s}^{\prime}$, como mostra o último termo da expressão (6).

4. Calcular a métrica $d_{k}$ que utiliza a distância de Hamming

$$
\left.d_{k}=\sum \mid \mathfrak{R}\left(y_{s}^{\prime}\right)-\mathfrak{R}\left(y_{s}^{\prime}\right) \cdot \operatorname{PRBS} 1\right) \mid
$$

sendo $d$ um vetor com quatro valores, respectivo às quatro configurações possíveis de pilotos espalhadas em cada símbolo OFDM, identificadas pela variável $k$.

5. Achar o valor do vetor $d$ mais próximo de zero usando a expressão

$$
v d_{i}=\min \left|d_{k}\right|
$$

sendo $-n<i<n$.

6. Ao terminar a análise das amostras, escolher o menor valor de $v d$.

7. Ajustar no tempo à posição do valor $v d$ anterior.

8. O mesmo procedimento pode ser aplicado em vários símbolos consecutivos a fim de se extrair um valor médio do ponto $v d$ alocado para o sincronismo.

\section{SIMULAÇÕES USANDO SINCRONIZADOR PROPOSTO}

Utilizando um modelo de simulação baseado no ISDB-T, foi implementado um módulo que simula o canal, onde são inseridos ruído AWGN, multicaminhos e desvios de freqüência. Além disso, em alguns casos, é inserido um atraso no sinal que simula a falta de sincronismo. Os parâmetros de simulação para o sinal de entrada são: modo 8k, IG 1/8, FEC 2/3, constelação 64-QAM, e $n=\mathrm{IG} / 2$ (conforme algoritmo de refinamento).

\section{A. TESTES COM RUÍDO GAUSSIANO BRANCO (AWGN)}

O comportamento da distância de Hamming $d$, para um sinal submetido a ruído AWGN e obedecendo a uma relação sinal ruído (SNR) de $18 \mathrm{~dB}$, é mostrado na Fig.12 a seguir. Como nenhum atraso foi utilizado para este caso, pode-se observar a eficiência da técnica para estas condições.

As partes do gráfico da Fig.12 sem informação correspondem a região de onde se tirou o intervalo de guarda, e não foi avaliada. Repetindo a simulação do sinal em 5 símbolos OFDM e variando-se a SNR, são encontrados os valores de $d$ mostrados na Fig. 13 .

Nesta parte, foram testados cinco símbolos OFDM continuamente, aumentando-se a SNR para se analisar o comportamento da função distância. A distância mínima é o ponto onde se inicia o símbolo, onde o intervalo de guarda tem mais semelhança ou menor distância de Hamming (próxima de zero). Mesmo com uma $\mathrm{SNR}=3 \mathrm{~dB}$ é possível um sincronismo dentro do intervalo de guarda, o que prova a grande robustez da técnica diante de canais AWGN.

\section{B. CANAIS BRASIL}

Foram testados no simulador os canais Brasil de A a E [18]. Neste caso, foram realizadas até 16 iterações, analisando-se até os 16 símbolos que chegariam inicialmente ao receptor, para testes de convergência e confiabilidade do algoritmo, como mostrado na Tabela I.

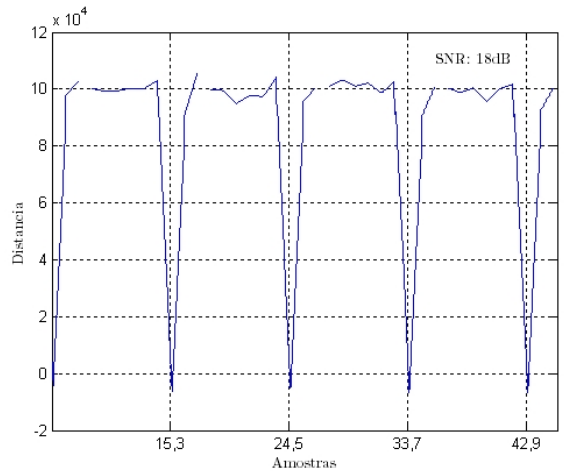

Fig.12. Comportamento da métrica baseada na distância de Hamming em condições de ruído gaussiano branco.

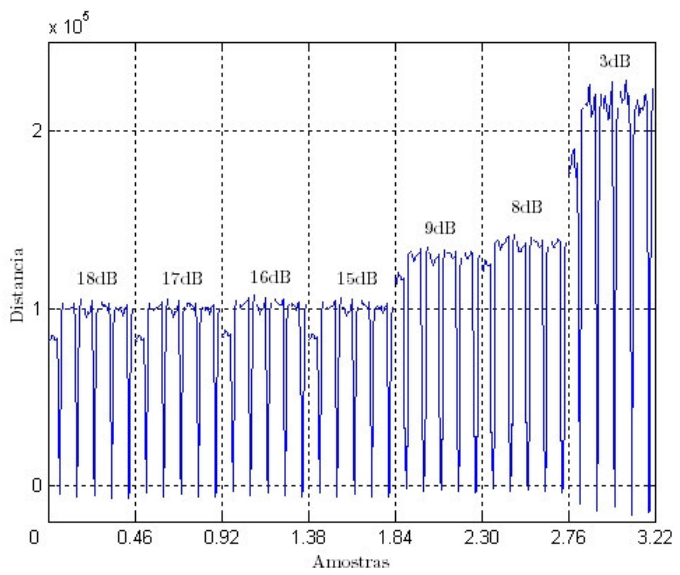

Fig.13. Comportamento da métrica variando-se intensidade de AWGN.

TABELA I

Comportamento da distância de Hamming para canais Brasil.

\begin{tabular}{|c|c|c|c|c|c|}
\hline \multirow{2}{*}{$\begin{array}{c}\text { No. de símbolos } \\
\text { avaliados }\end{array}$} & \multicolumn{5}{|c|}{ Erro médio em número de amostras para o } \\
\cline { 2 - 7 } canal & $\mathrm{A}$ & $\mathrm{B}$ & $\mathrm{C}$ & $\mathrm{D}$ & $\mathrm{E}$ \\
\hline 1 & 2 & 31 & 20 & 42 & 15 \\
\hline 8 & 0,07 & 8,00 & 6,35 & 2,4688 & 1,6719 \\
\hline 16 & 0,50 & 1,58 & 3,93 & 4,0800 & 0,1081 \\
\hline Erro máximo & 2 & 31 & 20 & 42 & 15 \\
\hline Erro mínimo & 0 & 1 & 2 & 1 & 0 \\
\hline
\end{tabular}

A tabela anterior apresenta erros muito pequenos, sendo que, como previsto, o maior erro é obtido na primeira iteração. $\mathrm{O}$ desempenho do sincronismo melhora a cada iteração, porém, em todos os casos, está dentro do intervalo de guarda, o que possibilita a recuperação do sinal sem perdas, através de técnicas de equalização. Por exemplo, 42 amostras erradas no Canal D, estão dentro do IG de 1152 amostras utilizado. Nesse caso, é importante considerar que foi encontrada a diferença de 42 amostras já que o caminho de maior potência está atrasado exatamente deste número de amostras, o que não produzirá perdas para os blocos subseqüentes do receptor. Assim, adotou-se neste trabalho apenas um símbolo para análise no sincronismo em freqüência já é o suficiente, o que torna este processo computacionalmente muito rápido. 
O comportamento para Canal E, característico para redes de freqüência única e que é enfatizado neste trabalho, também foi satisfatório. Como não existem atenuações dos ecos, o estimador alocou ora o sinal mais atrasado (com $1 \mu \mathrm{s}$ de atraso), equivalente a 15 amostras, ora o sinal principal sem atraso. Em qualquer dessas situações, será possível recuperar o sinal de vídeo.

\section{CANAL RAYLEIGH}

A recepção móvel é importante para aplicações multimídia portáteis ou receptores de carros. Para testar o sincronismo desses sinais, o simulador foi configurado com os parâmetros mostrados na Tabela II, com seis multipercursos e com Doppler variável seguindo um modelo Rayleigh, da mesma forma que trabalhado em [19]. Este canal é conhecido como GSM urbano. O resultado é mostrado na Fig. 14.

TABELA II

Canal de teste GSM urbano.

\begin{tabular}{|l|c|c|c|c|c|c|}
\hline & \multicolumn{7}{|c|}{ Caminhos } \\
\cline { 2 - 8 } & $\mathbf{1}$ & $\mathbf{2}$ & $\mathbf{3}$ & $\mathbf{4}$ & $\mathbf{5}$ & $\mathbf{6}$ \\
\hline Atraso $(\boldsymbol{\mu s})$ & 0 & 0,2 & 0,5 & 1,6 & 2,3 & 5 \\
\hline Atenuação $(\mathrm{dB})$ & 3 & 0 & 2 & 6 & 8 & 10 \\
\hline
\end{tabular}

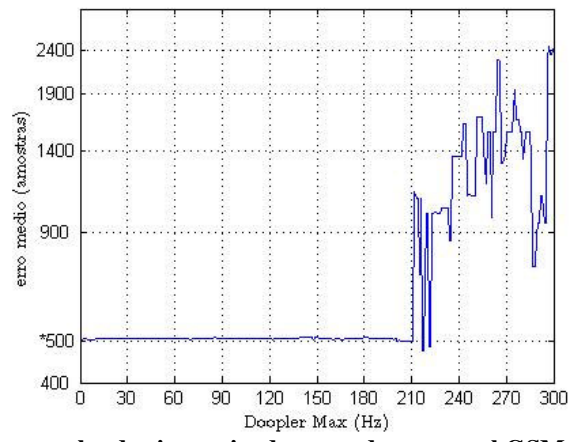

Fig.14. Desempenho do sincronizador usando um canal GSM urbano.

A curva de teste apresenta um comportamento bem próximo de 500 amostras, que é o atraso inserido nos testes. portanto o sincronismo, para esse caso, está garantido dentro do intervalo de guarda até um efeito Doppler de até $210 \mathrm{~Hz}$ de variação. Do mesmo modo, para recepção móvel além de um sincronizador, será necessário um estimador de canal Doppler ou um equalizador adaptativo.

\section{CONCLUSÕES}

Este trabalho discutiu algumas técnicas para se realizar o sincronismo de símbolo em sinais OFDM, e foi proposta uma nova técnica baseada em reconhecimento de padrões.

O sincronizador proposto teve comportamento satisfatório e, na grande maioria dos testes com multicaminho e desvios de frequiência, a posição encontrada está dentro do intervalo de guarda, apresentando complexidade muito baixa com número reduzido de símbolos para análise em relação aos métodos clássicos. Para o canal Brasil E, que representa um caso crítico de recepção em rede de freqüência única, o sincronizador mais uma vez se mostrou eficiente, alocando ora no sinal principal ora no eco de mesma amplitude.

\section{AGRADECIMENTOS}

Os autores gostariam de agradecer a CAPES, FAPESP e FAEP pelo apoio financeiro neste trabalho.

\section{REFERÊNCIAS}

[1] R. V. Nee, R. Prasad, "OFDM for Wirelles Multimídia Communications, Norwood", MA: Artech House Universal Personal Communication Library, 2000.

[2] T. Pollet, M. V. Bladel, and M. Moeneclaey, "BER sensitivity of OFDM systems to carrier frequency offset and wiener phase noise", IEEE Transactions on Communications, vol. 43, pp. 191-193, Fev-Abril de 1995.

[3] J.J. van de Beek, M. Sandell, M. Isaksson, P. O. Borjesson, "Lowcomplex frame synchronization in OFDM systems", Fourth IEEE International Conference on Universal Personal Communications, Pages: 982-986, Novembro de 1995.

[4] A. J. Coulson, "Maximum Likelihood Synchronization for OFDM Using a Pilot Symbol Analysis", IEEE Journal on Selected Areas in Communications, vol. 19, no. 12, dezembro de 2001.

[5] H. Minn, V. K. Bhargava, K. B. Letaief, "A Robust Timing and Frequency Synchronization for OFDM Systems", IEEE Transactions on Wireless Communications, Vol. 2, no. 4, Julho de 2003.

[6] Chi Chung Ko, Ronghong Mo, and Miao Shi, "A New Data Rotation Based CP Synchronization Scheme for OFDM Systems", IEEE Transaction on Broadcasting, vol. 51, no. 3, setembro de 2005.

[7] A. Palin and L. Rinne, "Symbol synchronization in OFDM system for time selective channel conditions", in Proc. of 1CECS'99, Pafos Cyprus, pp. 1581-1584., setembro de 1999.

[8] A. Palin and L. Rinne, "Enhanced symbol synchronization method for OFDM system in SFN channels", Global Telecommunications Conference - IEEE Globecom 98, Vol. 5, Page(s):2788 - 2793, Novembro de 1998.

[9] T. Schmidl, D. Cox, "Robust Frequency and Timing Synchronizatin for OFDM Systems", IEEE Communications letters, vol. 4, no. 7, pp 242-244, Julho de 2000

[10] P. H. Moose, "A Technique for Orthogonal Frequency Division Multiplexing Frequency Offset Correction", IEEE Transaction on Communications, Vol. 42, No. 10, pp.2908-2914, outubro de 1994.

[11] R. Duda, P. Hart, "Pattern Classification and Scene Analysis", Wiley-Interscience Publication, 1973.

[12] C. H. Chen, C. T. Chu, "Low complexity iris recognition based on wavelet probabilistic neural networks", IEEE International Joint Conference on Neural Networks (IJCNN 05), Vol.3, Pages:1930 - 1935, Agosto 2005.

[13] A. Abhyankar, L. Hornak, S. Schuckers, "Off-angle iris recognition using bi-orthogonal wavelet network system", Fourth IEEE Workshop on Automatic Identification Advanced Technologies, Pages: 239-244, Outubro de 2005.

[14] R. Larico, "Uma Proposta para Melhoria no Sistema de Reconhecimento de Íris Humana", Tese de Mestrado, FEEC-Unicamp, Janeiro de 2007.

[15] R. W. Hamming, "Error-detecting and error-correcting codes", Bell System Technical Journal 29(2):147-160, 1950.

[16] B. Y. Prasetyo, A. H. Aghvami, "Simplified frame structure for MMSE-based fast burst synchronisation in OFDM systems", Electronics Letters, vol. 35, pp. 617-618, Abril de 1999.

[17] Terrestrial Integrated Services Digital Broadcasting (ISDB-T) Document, "Specification of Channel Coding, Framing Structure and Modulation", 1998.

[18] SET/ABERT, "Digital Television Systems Brazilian Tests—Final Report Part 1," Anatel SP, Março 2000.

[19] G. Bedicks Jr, F. Yamada, F. Sukys, C. E. S. Dantas, L. T. M. Raunheitte, C. Akamine, "Results of the ISDB-T system tests, as part of digital TV study carried out in Brazil", IEEE Transactions on Broadcasting, Volume 52, Issue 1, Page(s): 38 - 44, Março de 2006. 